\title{
Use of amiodarone in bradycardia-tachycardia syndrome
}

\author{
ALLEN K. BROWN, ROBERT A. PRIMHAK, AND PAUL NEWTON \\ From the Royal Lancaster Infirmary, Lancaster
}

SUMMARY Five patients with the bradycardia-tachycardia syndrome have been treated successfully with the antiarrhythmic agent amiodarone. Three patients were treated for over nine months and one of these patients had corneal micro deposits. One patient had to be taken off the drug because of side effects. Amiodarone should be tried in patients suffering from the bradycardia-tachycardia syndrome before resorting to cardiac pacing.

Bradycardia-tachycardia syndrome usually reflects the presence of sinoatrial disease, in which episodes of supraventricular tachycardia complicate sinus bradycardia with or without periods of sinus arrest or sinoatrial block.

Dizziness, syncope, or convulsions may result from cerebral ischaemia secondary to bradycardia, and tachycardia may cause palpitation, ischaemic cardiac pain, dyspnoea, and, more rarely, hypotension and cardiac failure. Systemic emboli may occur particularly in patients over 50 years (Rubenstein et al., 1972; Ferrer, 1973).

The aetiology of the sick sinus syndrome and the bradycardia-tachycardia syndrome is often unknown, but associations with coronary artery disease, thyrotoxicosis, cardiomyopathy, amyloidosis, diahetes, and cardiac surgery have been reported, and there is a rare familial form (British Medical Fournal, 1977).

Correlation of electrocardiographic rhythm changes with symptoms over 24-hour periods using ambulatory monitoring equipment facilitates diagnosis of the syndrome, and the combination of implanted pacemakers and appropriate antiarrhythmic agents has been successful in controlling the clinical features. Drug treatment alone is difficult since agents which control the tachycardia may aggravate the bradycardia and vice versa. The ideal drug would be effective in controlling bradycardia and tachycardia without the need for cardiac pacing, and in this communication we report the results of treating 5 patients with the antiarrhythmic agent amiodarone.

Received for publication 30 September 1977

\section{Case reports}

\section{CASE 1}

A 54-year-old man was admitted in September 1974 with an acute anterior myocardial infarction complicated by ventricular fibrillation and atrial fibrillation. He had received methyldopa for 6 years previously for essential hypertension. In June 1976, he was readmitted with bouts of ischaemic cardiac pain associated with periods of rapid atrial fibrillation followed by sinus bradycardia at 30 beats a minute. Treatment with digoxin, verapamil, and propranolol in turn caused sinus bradycardia associated with faintness and dizziness, and failed to prevent periods of atrial fibrillation.

Amiodarone $200 \mathrm{mg}$ twice daily was started in July 1976, and was subsequently reduced to $100 \mathrm{mg}$ twice daily because of nausea. He has had no further bouts of chest pain, dizziness, faintness, or palpitation and has now taken the drug for 13 months. Corneal micro deposits were noted 5 months after starting amiodarone, but have not increased since, and have not interfered with vision. The condition was explained to the patient who expressed a desire to continue with the drug. A 24-hour electrocardiographic recording using the Oxford Medilog equipment showed no arrhythmia one year after the start of treatment.

\section{CASE 2}

A 73-year-old man was admitted to hospital in July 1976 with a 3-month history of retrosternal pain variably related to exertion. For 2 days before admission he had suffered from repeated episodes of dizziness and inability to concentrate, and on one 
occasion had lost consciousness resulting in a fall and a scalp laceration. He had been treated with guanethidine and a thiazide diuretic for two years previously.

On admission his blood pressure was $180 / 100$ $\mathrm{mmHg}$, with a basal midsystolic murmur and the electrocardiogram showed left ventricular hypertrophy and prolonged bouts of atrial tachycardia at 150 beats a minute, and sinus bradycardia at 40 beats a minute. The slow rhythm was associated with dizziness and feelings of syncope.

Digoxin caused persistent sinus bradycardia and verapamil was subsequently tried without altering the frequency of the tachycardia and bradycardia. Amiodarone $200 \mathrm{mg}$ twice daily successfully controlled the symptoms, and routine, regular 12 lead electrocardiograms have shown sinus rhythm. A 24hour electrocardiogram in June 1976 shows sinus rhythm with a rate which drops to 46 a minute on occasions with no symptoms.

\section{CASE 3}

A 48-year-old woman was investigated in September 1976 for a pyrexial illness. Chest $x$-ray film showed diffuse pulmonary infiltration, and the ESR was $50 \mathrm{~mm} /$ hour (Westergren). A diagnosis of allergic alveolitis was made, and she was treated with prednisolone. In December 1976 she was admitted because of transient loss of consciousness, and a routine electrocardiogram showed the features of the bradycardia-tachycardia syndrome with sinus bradycardia at 30 beats a minute coinciding with transient loss of consciousness. A trial of propranolol caused extreme bradycardia and because of previous success in this condition, amiodarone $200 \mathrm{mg}$ twice daily was started and sinus rhythm was restored, with no further loss of consciousness.

Six weeks later the amiodarone was withdrawn without return of symptoms and she has remained well subsequently.

\section{CASE 4}

A man aged 87 years was admitted in October 1976 with a 6-week history of transient disturbance of consciousness and short periods of lower sternal tight feelings. A 24-hour electrocardiogram using the Oxford Medilog system showed periods of supraventricular tachycardia at 140 per minute associated with retrosternal discomfort, and periods of sinus bradycardia at 40 a minute coinciding with disturbance of conscious level (see Fig.). Treatment with phenytoin made no difference to the clinical features and amiodarone $200 \mathrm{mg}$ twice daily was started 3 weeks later with complete abolition of symptoms during subsequent 9 months on the drug.
CASE 5

A woman aged 47 years was first seen in 1970 with paroxysmal supraventricular tachycardia occurring particularly at night. The patient reported pulse rates at under 40 a minute on other occasions. She was treated unsuccessfully with courses of propranolol, phenytoin, and procainamide, and periods of sinus bradycardia were seen to be associated with exhaustion and feelings of faintness. In January 1977, her blood pressure was $125 / 80$ and a 24 -hour electrocardiogram showed periods of sinus tachycardia at 130 a minute alternating with sinus bradycardia at 32 a minute and sinus arrest. She was started on amiodarone $200 \mathrm{mg}$ twice daily, with no improvement, but on $200 \mathrm{mg}$ three times per day she had no cardiac symptoms, and a repeat 24-hour electrocardiogram showed sinus rhythm varying between 60 and 84 beats a minute. She complained of feeling unwell without specific features, and blood pressure rose to $190 / 125 \mathrm{mmHg}$. Amiodarone was stopped after 10 weeks but the blood pressure remained high, and she has been on hypotensive agents since. The palpitation and exhaustion associated with bradycardia have persisted, and she is awaiting permanent cardiac pacemaking.

\section{Discussion}

Amiodarone is a benzofuran derivative which was originally introduced as an antianginal agent, but has subsequently been shown to be valuable in the management of atrial and ventricular arrhythmias and in the Wolff-Parkinson-White syndrome (Rosenbaum et al., 1976). There have been no previous reports of the use of amiodarone in the bradycardia-tachycardia syndrome.

The principal actions of amiodarone are to cause relaxation of vascular smooth muscle resulting in a slight fall in systemic vascular resistance with slight reduction in systemic blood pressure, and a fall in coronary arterial resistance and improved coronary blood flow. The drug also causes an atropineresistant bradycardia, and reduces the effects of catecholamines and sympathetic stimulation without acting as a competitive $\alpha$ and $\beta$ receptor blocking agent. Singh and Vaughan Williams (1970) report that amiodarone causes prolongation of the duration of the action potential in atrial and ventricular muscle with no change in the resting potential, and amiodarone has been grouped with bretylium as a class 3 antiarrhythmic agent (Olsson et al., 1973; Singh and Hauswirth, 1974). The net haemodynamic effects of the drug are an increase in coronary arterial blood flow, and reduction in left ventricular work and in myocardial oxygen consumption owing to the slowing of the heart and to 


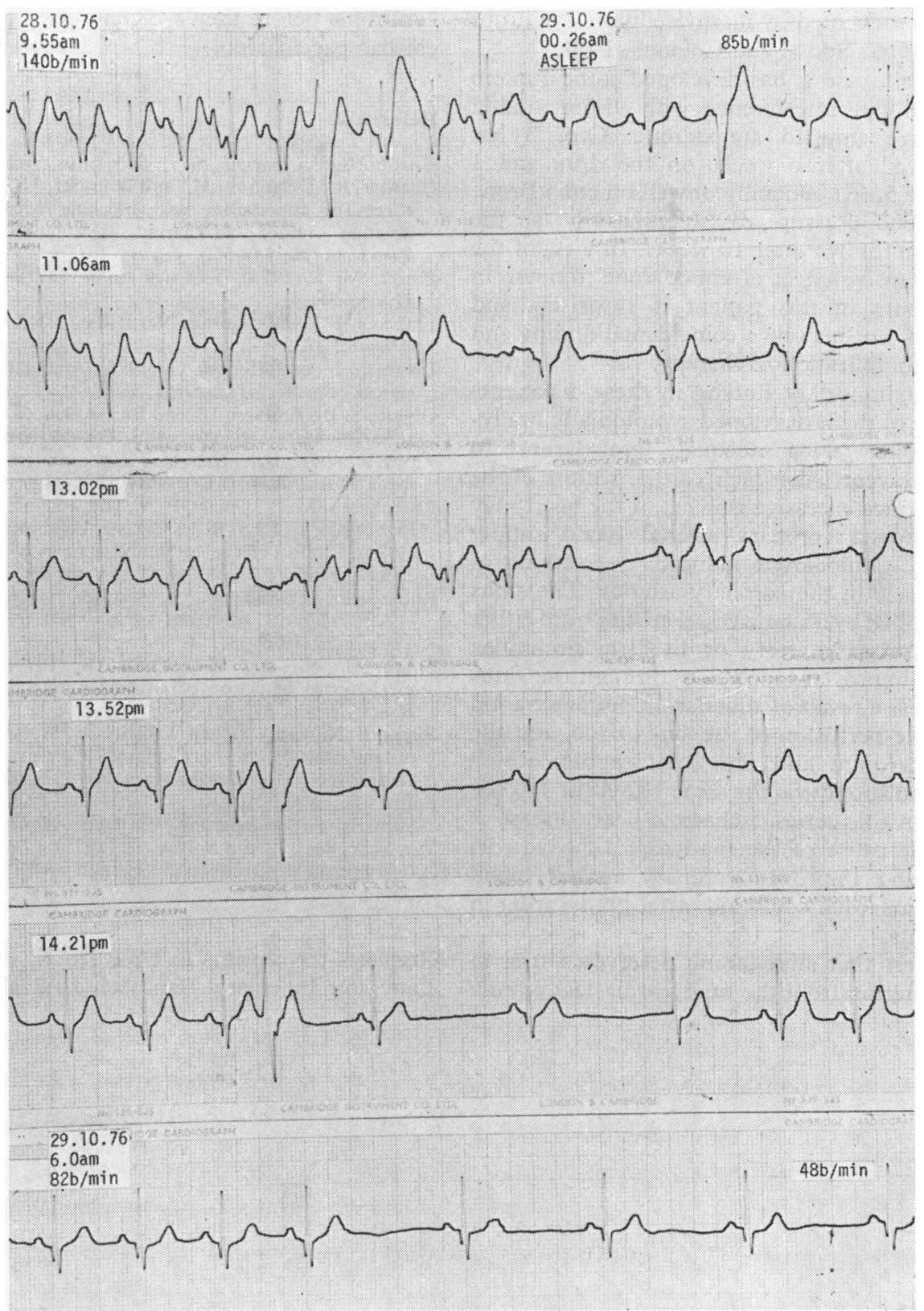

Fig. Twenty-four hour electrocardiogram from case 4 showing supraventricular tachycardia and sinus bradycardia.

the reduction in systemic vascular resistance (Charlier et al., 1972).

There is ample evidence of the efficacy of amiodarone in the management of arrhythmias, particularly of supraventricular origin (Van Schepdael and Solvay, 1975; Rosenbaum et al., 1976), but its use has been limited because of concern over side effects. Bradycardia, thyroid disorders, skin lesions, photosensitivity, and one case of sensory ataxic paraesthesiae and tremor have been described (Lustman and Monsea, 1974), but most concern has centred on the frequency of corneal micro deposits. The deposits, however, are usually found below the pupil, do not interfere with vision, are reversible, 
and may be prevented by methylcellulose eye drops (Francois, 1969; Soussi and Colonna, 1974).

One patient, case 1 , has developed corneal micro deposits without interference with vision, and 2 patients have stopped amiodarone. Case 3 has remained well after 6 weeks on the drug and 1 patient, case 5, felt generally unwell on amiodarone and her blood pressure rose significantly, so the drug was withdrawn after 10 weeks. In view of the usual hypotensive effect of amiodarone, the rise in blood pressure in this patient is surprising and unexplained, but may be a coincidental finding and not a true complication of therapy.

The most important finding in these 5 patients is that none of them developed symptomatic bradycardia despite taking adequate amiodarone to prevent supraventricular tachycardia. Failure of the drug to produce excessive slowing of the heart may reflect improved coronary arterial blood supply because of amiodarone-induced relaxation of smooth muscle in the coronary arteries. The sinus node is supplied by a single vessel arising from either the right coronary artery or the left circumflex artery, and improved blood flow through the sinus node artery as a result of amiodarone administration may improve perfusion of the sinus node, and the atrial myocardium and interatrial septum which receive blood supply via the same vessel. In 3 of the 5 patients in this series, ischaemic heart disease is the probable cause of the bradycardia-tachycardia syndrome and improved coronary arterial blood flow by amiodarone could aid atrial bradycardia in these patients.

We suggest that amiodarone deserves a trial in patients suffering from the bradycardia-tachycardia syndrome before they are committed to permanent cardiac pacemaking.

\section{References}

British Medical fournal (1977). Sick sinus syndrome. 1, 4-5.

Charlier, R., Delaunois, G., and Bauthier, J. (1972). Opposite effects of amiodarone and blocking agents on cardiac function under adrenergic stimulation. Arzneimittel Forschung, 22, 545-552.

Ferrer, M. I. (1973). The sick sinus syndrome. Circulation, 47, 635-641.

Francois, J. (1969). Cornea Verticillata. Documenta Ophthalmologica, 27, 235-250.

Lustman, F., and Monsea, G. (1974). Amiodarone and neurological side effects. Lancet, 1, 568 .

Olsson, S. B., Brorson, L., and Varnauskas, E. (1973). Class 3 antiarrhythmic action in man. Observations from monophasic action potential recordings and amiodarone treatment. British Heart fournal, 35, 1255-1259.

Rosenbaum, M. B., Chiale, P. A., Halpern, M. S., Nau, G. J., Przybylski, J., Levi, R. J., Lazzari, J. O., and Elizari, M. V. (1976). Clinical efficacy of amiodarone as an antiarrhythmic agent. American fournal of Cardiology, 38, 934-944.

Rubenstein, J., Schulman, C., Yurchak, P., and DeSanctis, R. (1972). Clinical spectrum of the sick sinus syndrome. Circulation, 46, 5-13.

Singh, B. N., and Hauswirth, O. (1974). Comparative mechanisms of action of antiarrhythmic drugs. American Heart fournal, 87, 367-382.

Singh, B. N., and Vaughan Williams, E. M. (1970). The effect of amiodarone, a new anti-anginal drug, on cardiac muscle. British fournal of Pharmacology, 39, 657-667.

Soussi, A., and Colonna, D. (1974). Troubles du rythme auriculaire et amiodarone. Fournal des Agregés, 7, 43.

Van Schepdael, J., and Solvay, H. (1975). L'amiodarone dans le traitment des troubles du rythme cardiagne. Praxis, 64, 30-34.

Requests for reprints to Dr Allen K. Brown, Royal Lancaster Infirmary, Lancaster LA1 4RP. 